\title{
Investigating How the Degree of Radiological Correction Corresponds to Patient Reported Outcomes in Scarf Osteotomy
}

\author{
Karam Ahmad ${ }^{1 *}$ and Anand Pillai ${ }^{2}$ \\ ${ }^{1}$ Imperial College NHS Trust, UK \\ ${ }^{2}$ University Hospital of South Manchester, UK \\ *Corresponding author: Karam Ahmad, Imperial College NHS Trust, Praed St, London, UK
}

Submission: 恝 October 02, 2017; Published: 眥June 13, 2018

\begin{abstract}
Background: Patient-reported outcome measures (PROMs) are important in modern healthcare systems. Previous studies on PROMs in halluxvalgus (HV) surgery show positive outcomes. We investigated the relationship between radiological correction and PROMs in hallux-valgus surgery.

Patients and methods: A total of 350 patients underwent foot and ankle surgery and signed up to the PROMS 2.0 scheme. 97 patients provided complete pre-operative and post-operative data of which 40 had undergone scarf osteotomy and fitted in to selection criteria. Data collection was performed using the Manchester-Oxford Foot Questionnaire (MOxFQ). Radiological measurements (HVA-hallux-valgus angle and IMTA- Intermetatarsal angle) were calculated by two independent blinded foot and ankle surgeons on PACS. A comparative analysis was done between degree of radiological correction and pre-/post-op PROMs. Statistical tests were carried out using IBM-SPSS Statistics (V19).
\end{abstract}

Results: 40 patients included with female predominance, equal side distribution and no bilateral procedures. Average age at time of surgery- 60.7 years (Range 29-88). Mean pre-op MOxFQ=52.9 (12.0-100.0), mean post-op MOxFQ=23.6 (0-91.3) and mean improvement in MOxFQ=29.4 (-18.7-73.3) $\mathrm{p}<0.05$. HVA- average pre-op angle $=34.97^{\circ}\left(13.20^{\circ}-57.00\right)$, average post-op angle $=15.01^{\circ}(2.00-39.10)$. Mean $\mathrm{HV}$ correction angle $=19.97(3.20-47.50)$. There was statistically significant correlation between pre-op/post-op HVA measurements and pre-op/post-op PROMS values respectively ( $<<0.05$ ). There was direct correlation between HVA correction and improvement in PROMS

For IMTA, average pre-op angle $=17.38^{\circ}(10.40-27.60)$, average post-op angle $=12.09^{\circ}(6.00-21.10)$. Mean IMTA Correction=5.29(0.20-15.80). There was statistically significant correlation between pre-op/post-op IMTA measurements and pre-op/post-op PROMS values respectively ( $\mathrm{p}<0.05)$. There was direct correlation between IMTA correction and improvement in PROMS.

Conclusion: Hallux-valgus surgery is an effective procedure with high PROMS. There is a positive correlation between PROMS and degree of deformity. As HVA/IMTA increase, PROMs decrease. As HVA and IMTA correction increases, PROMs improves. Better surgical correction leads to better PROMS.

Keywords: PROMs; Foot and ankle; Hallux valgus; Scarf osteotomy; Hallux valgus angle; Inter-metatarsal angle

\section{Introduction}

Patient reported outcome measures (PROMs) form a fundamental basis of 21 st century health care systems. It is a Department of Health (DoH) based national requirement to collect PROMs [1]. PROMs allow us to take another perspective on surgical outcomes. They can be used both alongside and comparatively with more objective perspectives [2]. PROMs allow us to move towards a more patient centric healthcare service.

Scarf osteotomy has been known to produce very good outcomes in treating hallux valgus [3]. Previous studies show that PROMs are very positive. With a higher satisfaction in older age groups and a slightly superior improvement in male patients [4]. Objectively, scarf osteotomy is often described as safe, giving better aesthetic and functional results than other options for hallux valgus treatment. It is also associated with early recovery and return to work [5].

We look to investigate the relationship between PROMs and correction angles in hallux valgus patients undergoing scarf osteotomy. PROMs will be assessed using the Manchester- Oxford foot questionnaire, a 16-item patient reported outcome-measuring tool. This has been validated for use in foot and ankle surgery [6]. We used hallux valgus angles, and inter-metatarsal angles to 
determine the relationship between correction angles and patient reported outcome improvement.

\section{Patients and Methods}

This is a prospective study looking at patients presenting with symptomatic hallux valgus. Patients reported their outcomes using a semi-automated email based system- Amplitude ${ }^{\mathrm{Tm}}$. Patients were signed up to the PROMs2.0 scheme, which was set up at various trusts across the North West of England. Patients undergoing foot and ankle procedures were asked to participate at their preop appointments. Once signed up, they would be included in the study, and sent prompts at regular intervals, pre-operatively, and post op 6 months to fill in the above questionnaires. The method of communication was by email.

Inclusion criteria- Patients were required to be fit for general anesthesia, and must have returned pre-operative, and post operative questionnaires. Overall we had 350 patients who took part in the PROMs2.0 study. This consisted of various foot and ankle procedures, 97 patients responded out of the 350 with preoperative and post operative PROMs data (27.7\% participation). Of this 97,40 patients had undergone scarf osteotomy and these were the patients who were included in this study. Therefore they were not consecutive cases. There was no power calculations involved.

All procedures were part of a single unit series done between 3 fellowship trained foot and ankle surgeons. Surgery was performed as a day case procedure. All patients had GA with a local block. This was a medial approached standard procedure with soft tissue release via a separate interspace incision of $1.5 \mathrm{cms}$. Prophylactic antibiotics were given in accordance with the local anti-microbial policy. Weight bearing was allowed post operatively in a heel wedge shoe (DARCO) and all patients were seen at 2 weeks post op for a wound check. At this point patients were advised to maintain active foot and ankle movement. At six weeks physiotherapy was commenced for patients based on radiological and clinical evidence of healing. Regular follow up occurred at intervals of 3, 6 and 12 months (part of study protocol)

Hallux valgus and inter-metatarsal angles were calculated by two independent blinded foot and ankle surgeons on PACs. Based on average pre-operative HV/IMT angles and average post-operative HV/IMT angles- a correction angle was calculated. Comparative analysis was done based upon these correction angles and changes in PROMs.

Statistical tests carried out were done using the program IBM SPSS Statistics (2015) IBM, New York. A p value of 0.05 was considered to be significant giving us $95 \%$ confidence limits in our data. We used T-tests to assess the two independent data sets.

\section{Results}

40 patients underwent the procedure; 21 were right sided, and 19 left. There were 35 Female and 5 male patients. Average age was 60.7 years (range 29-88yrs). There were no bilateral procedures in our cohort. With regards to HV angles, the average pre-operative angle was $34.97^{\circ}$ with a range of $13.2-57.0^{\circ}$. Average post-operative angle was $15.01^{\circ}$ with a range of $2.00-39.10^{\circ}$. Average correction angle was $19.97^{\circ}$ with a range of 3.20 to $47.50^{\circ}$.

With regards to IMT angles, the average pre-operative angle was $17.38^{\circ}$ with a range of $10.40-27.60^{\circ}$. Average post-operative angle was $12.09^{\circ}$ with a range of $6.00-21.10^{\circ}$. Average correction angle was $5.29^{\circ}$ with a range of 0.20 to $15.80^{\circ}$. PROMs results were as follows- the average pre-operative $\mathrm{MOXFQ}$ was 52.9 with a range of 12.0 to 100.0. Average post-operative MOxFQ was 23.6 with a range of 0.0-91.3. Average change in MOxFQ was 29.4 with a range of -18.7 to 73.3 . The raw results are tabulated in Table $1 \& 2$, and graphical representation of corresponding data in Figure 1-3.

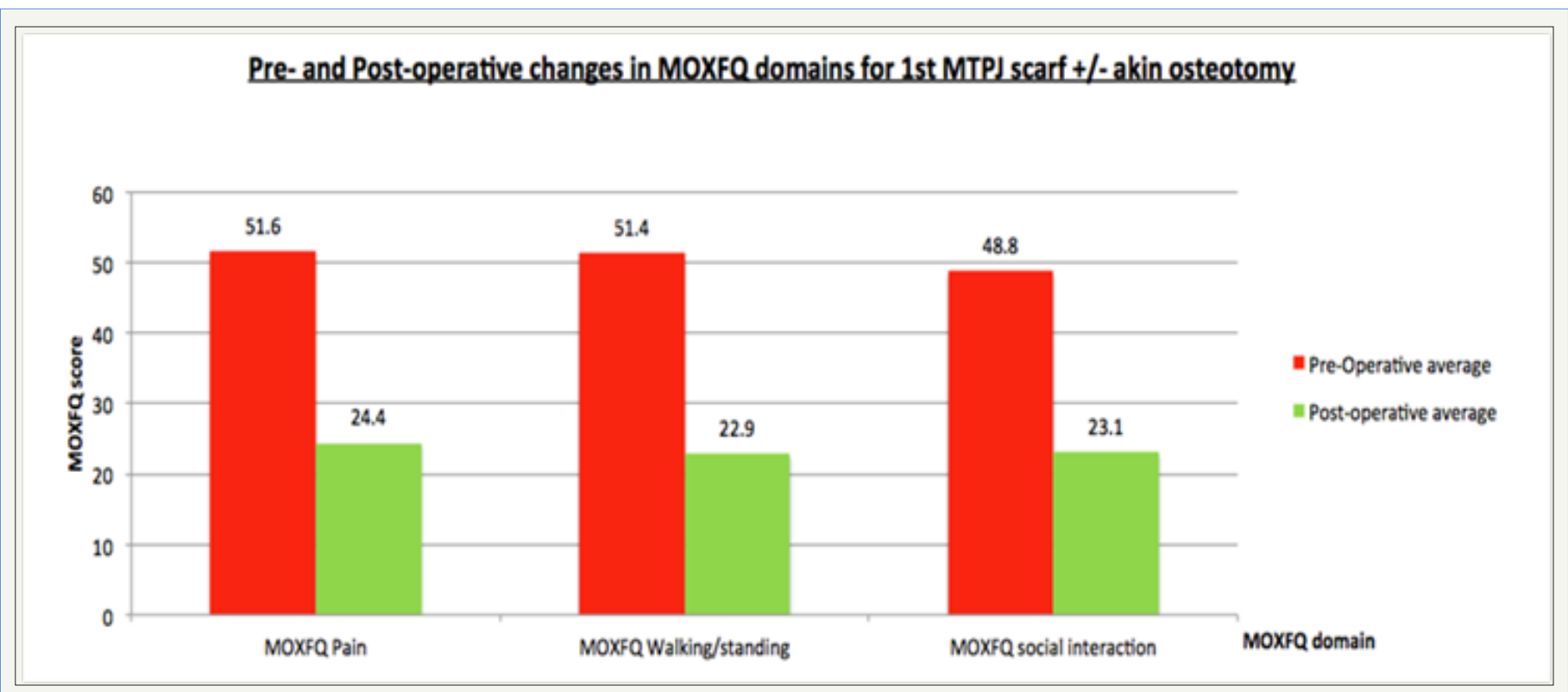

Figure 1 


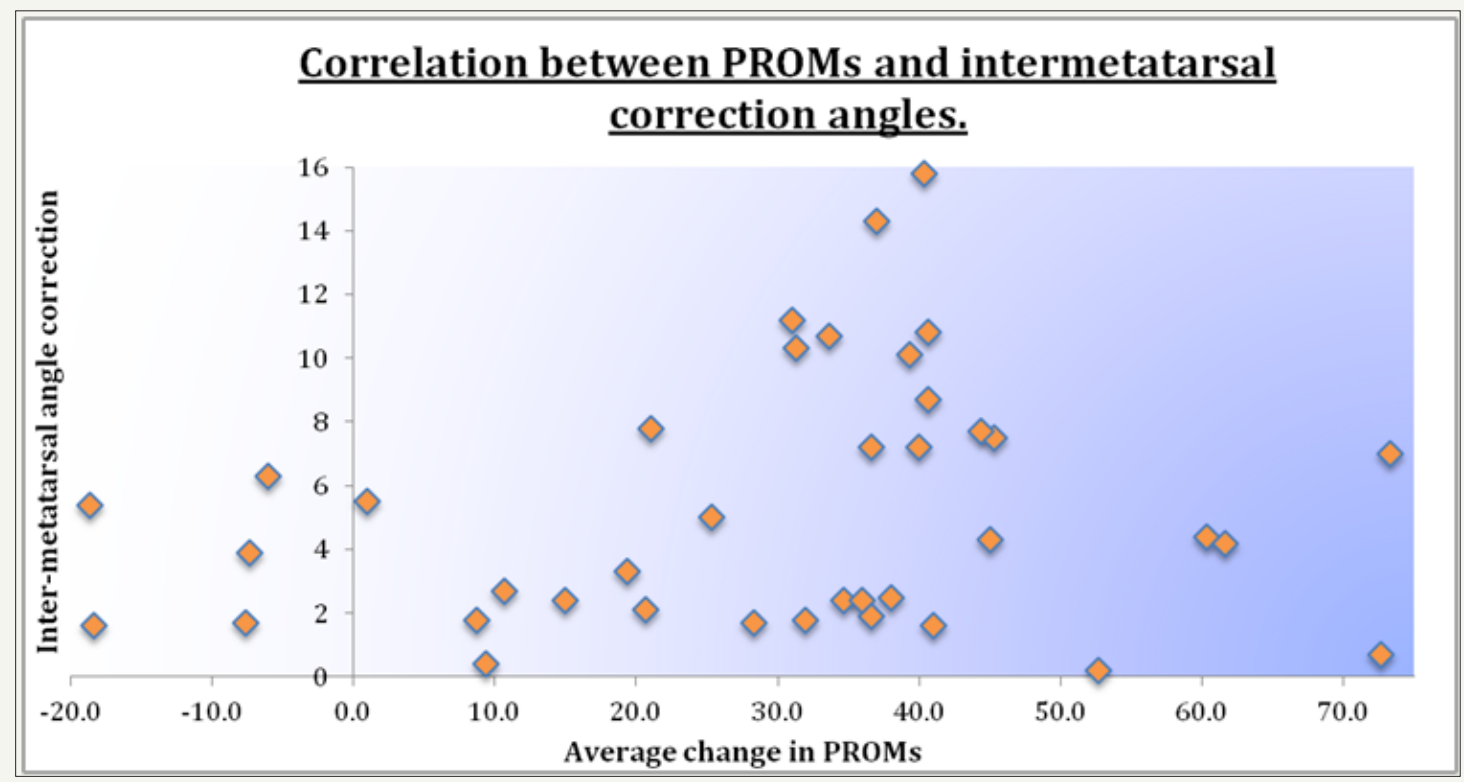

Figure 2

Table 1

\begin{tabular}{|c|c|c|c|}
\hline & Pre-OP Score & Post-0P Score & P value (<0.05) \\
\hline Pain-average (range) & $51.6(5-100)$ & $24.4(0-100)$ & $<0.00001$ \\
\hline Walking/standing average (range) & $51.4(0-96)$ & $22.9(0-86)$ & $<0.00001$ \\
\hline Social interaction average (range) & $48.8(0-100)$ & $23.1(0-88)$ & $<0.00001$ \\
\hline
\end{tabular}

Table 2

\begin{tabular}{|c|c|c|c|c|c|c|c|c|c|}
\hline Patient & $\begin{array}{l}\text { Pre-Op Hv } \\
\text { Angle }\end{array}$ & $\begin{array}{l}\text { Post-Op Hv } \\
\text { Angle }\end{array}$ & $\begin{array}{c}\mathrm{Hv} \\
\text { Correction } \\
\text { Angle }\end{array}$ & $\begin{array}{l}\text { Pre-0p Imt } \\
\text { Angle }\end{array}$ & $\begin{array}{l}\text { Post Op Imt } \\
\text { Angle }\end{array}$ & $\begin{array}{c}\text { Imt } \\
\text { Correction } \\
\text { Angle }\end{array}$ & $\begin{array}{c}\text { Average } \\
\text { Pre-0p Mox } \\
\text { fq }\end{array}$ & $\begin{array}{c}\text { Average } \\
\text { Post-OP } \\
\text { Mox fq }\end{array}$ & $\begin{array}{c}\text { Average } \\
\text { Correction } \\
\text { of Mox fq }\end{array}$ \\
\hline 1 & 47.7 & 39.1 & 8.6 & 21.5 & 21.1 & 0.4 & 73.3 & 64.0 & 9.3 \\
\hline 2 & 39.7 & 2.0 & 37.7 & 20.7 & 14.4 & 6.3 & 60.7 & 66.7 & -6.0 \\
\hline 3 & 25.7 & 7.0 & 18.7 & 27.6 & 13.3 & 14.3 & 37.0 & 0.0 & 37.0 \\
\hline 4 & 30 & 9.0 & 21.0 & 15.9 & 12.6 & 3.3 & 43.0 & 23.7 & 19.3 \\
\hline 5 & 46.8 & 19.6 & 27.2 & 22.3 & 15.1 & 7.2 & 48.3 & 8.3 & 40.0 \\
\hline 6 & 24.2 & 6.1 & 18.1 & 21.1 & 10.8 & 10.3 & 67.7 & 36.3 & 31.3 \\
\hline 7 & 44.0 & 20.0 & 24.0 & 18.3 & 13.9 & 4.4 & 67.7 & 7.3 & 60.3 \\
\hline 8 & 13.2 & 10.0 & 3.2 & 17.1 & 12.8 & 4.3 & 63.3 & 18.3 & 45.0 \\
\hline 9 & 44.7 & 23.1 & 21.6 & 22.1 & 15.1 & 7.0 & 73.3 & 0.0 & 73.3 \\
\hline 10 & 39.7 & 11.6 & 28.1 & 24.4 & 8.6 & 15.8 & 66.3 & 26.0 & 40.3 \\
\hline 11 & 22.7 & 12.7 & 10.0 & 18.3 & 13.3 & 5.0 & 37.7 & 12.3 & 25.3 \\
\hline 12 & 51.8 & 22.8 & 29.0 & 20.3 & 12.8 & 7.5 & 55.3 & 10.0 & 45.3 \\
\hline 13 & 25.1 & 11.9 & 13.2 & 16.8 & 15.0 & 1.8 & 100.0 & 91.3 & 8.7 \\
\hline 14 & 22.3 & 11.8 & 10.5 & 12.8 & 11.0 & 1.8 & 46.7 & 14.7 & 32.0 \\
\hline 15 & 21.0 & 10.0 & 11 & 12.6 & 10.2 & 2.4 & 38.7 & 4.0 & 34.7 \\
\hline 16 & 24.4 & 12.9 & 11.5 & 22.9 & 15.1 & 7.8 & 54.3 & 33.3 & 21.0 \\
\hline 17 & 25.9 & 21.4 & 4.5 & 17.3 & 17.5 & -0.2 & 52.7 & 0.0 & 52.7 \\
\hline 18 & 47 & 25.8 & 21.2 & 13.2 & 12.5 & 0.7 & 78.0 & 5.3 & 72.7 \\
\hline 19 & 42.1 & 15.8 & 26.3 & 15.4 & 10.0 & 5.4 & 38.0 & 56.7 & -18.7 \\
\hline
\end{tabular}




\begin{tabular}{|c|c|c|c|c|c|c|c|c|c|}
\hline 20 & 49.1 & 14.0 & 35.1 & 15.3 & 9.8 & 5.5 & 32.0 & 31 & 1.0 \\
\hline 21 & 54.0 & 16.0 & 38.0 & 20.1 & 9.3 & 10.8 & 47.7 & 7.0 & 40.7 \\
\hline 22 & 26.4 & 13 & 13.4 & 14.1 & 12.4 & 1.7 & 28.3 & 0.0 & 28.3 \\
\hline 23 & 29.1 & 16.3 & 12.8 & 14.9 & 12.4 & 2.5 & 62.0 & 24.0 & 38.0 \\
\hline 24 & 28.9 & 16.3 & 12.6 & 14.0 & 11.9 & 2.1 & 27.3 & 6.7 & 20.7 \\
\hline 25 & 43.2 & 30 & 13.2 & 26.2 & 15.0 & 11.2 & 98.7 & 67.7 & 31.0 \\
\hline 26 & 54.7 & 16.9 & 37.8 & 20.9 & 10.2 & 10.7 & 52.7 & 19.0 & 33.7 \\
\hline 27 & 21.0 & 10.0 & 11.0 & 12.2 & 9.8 & 2.4 & 29.0 & 14.0 & 15.0 \\
\hline 28 & 45.8 & 11.1 & 34.7 & 19.3 & 9.2 & 10.1 & 44.7 & 5.3 & 39.3 \\
\hline 29 & 34.6 & 7.6 & 27.0 & 14.7 & 6.0 & 8.7 & 40.7 & 0.0 & 40.7 \\
\hline 30 & 21.4 & 15.0 & 6.4 & 13.4 & 9.5 & 3.9 & 41.3 & 48.7 & -7.3 \\
\hline 31 & 57.0 & 11.2 & 45.8 & 17.3 & 14.6 & 2.7 & 12.0 & 1.3 & 10.7 \\
\hline 32 & 32.3 & 18.0 & 14.3 & 10.4 & 8.8 & 1.6 & 41.0 & 0.0 & 41.0 \\
\hline 33 & 20.0 & 13.3 & 6.7 & 12.2 & 10.3 & 1.9 & 79.3 & 42.7 & 36.7 \\
\hline 34 & 38.5 & 12.5 & 26 & 13.0 & 11.4 & 1.6 & 13.0 & 31.3 & -18.3 \\
\hline 35 & 34.5 & 15.5 & 19.0 & 20.3 & 13.1 & 7.2 & 89.0 & 52.3 & 36.7 \\
\hline 36 & 52.7 & 5.2 & 47.5 & 17.2 & 9.5 & 7.7 & 52.0 & 7.7 & 44.3 \\
\hline 37 & 17.0 & 13.7 & 3.3 & 10.6 & 8.9 & 1.7 & 58.0 & 65.7 & -7.7 \\
\hline 38 & 35.0 & 15.0 & 20 & 17.4 & 12.1 & 5.3 & 52.9 & 23.6 & 29.4 \\
\hline 39 & 21.0 & 9.7 & 11.30 & 12.7 & 10.3 & 2.4 & 52.0 & 16.0 & 36.0 \\
\hline 40 & 44.7 & 27.3 & 17.40 & 18.6 & 14.4 & 4.2 & 61.7 & 0.0 & 61.7 \\
\hline Average & 34.97 & 15.01 & 19.97 & 17.38 & 12.1 & 5.28 & 52.93 & 23.56 & 29.38 \\
\hline Range L & 13.2 & 2.0 & 3.20 & 10.4 & 6.0 & -0.2 & 12.0 & 0.0 & -18.67 \\
\hline Range H & 57.0 & 39.1 & 47.5 & 27.6 & 21.1 & 15.8 & 100 & 91.33 & 73.33 \\
\hline
\end{tabular}

\section{Discussion}

PROMs developed in 2009 [1], as part of a department of health initiative become especially important where survival is not as important as is quality of life. Our study in 2015, presented at BOFAS, showed that hallux valgus is an effective procedure, providing good outcomes [4].

In this study, we looked at PROMs- namely MOxFQ and the effect hallux-valgus and inter-metatarsal angles have on them. We focused on MOXFQ as it is a scoring system that has been used in various studies, one described it as a tool with 'good measurement properties...for hallux valgus' MOxFQ has been validated for use in foot and ankle surgery. The specificity of MOxFQ with regards to foot and ankle surgery makes it ideal. However one criticism is that it fails to look at the patient's general wellbeing beyond the foot.

Our studies suggest that trends exist between degree of deformity and PROMs. We have found that as the level of preop deformity increased, the level of patient function/outcomes worsened. This shows a direct correlation between amount of deformity and functional scores. A correlation, which is statistically significant.

Furthermore, our results suggest that as the degree of correction increases, the patient reported outcome scores also improve. This is the first study, which shows that the amount of surgical correction achieved- measured using radiological parameters, translates to real time functional improvement. This is evidenced in our independent PROMs data collection. These results enable us to assess how degree of deformity can impact patient reported outcomes. It suggests that the greatest correction, leading to the most anatomically correct positioning leads to the greatest satisfaction amongst patients.

The limitations of our study are that we had a small cohort of patients. We lost many patients due to a lack of participation in PROMs 2.0. There was $27.7 \%$ participation in the study. We have discussed loss of patients in previous studies, in essence we can improve participation by offering patient choice of online/paper versions of the questionnaire, increasing reminder messages sent to patients when scores are due and getting patients to fill in the questionnaire in clinics. A greater number of patients would help make the results more reliable. It is prudent to note a very small proportion of patients did not feel improvement in outcomes despite improved radiological correction $[7,8]$.

\section{Conclusion}

We can deduce the following conclusions from our study, firstly, that the level of deformity in hallux valgus can affect functionality of patients and reported outcomes. Secondly, HV surgery results in improved functional scores, and finally that one must ensure effort is made for complete restoration of normal anatomy to enable optimum function for sufferers of hallux valgus. 


\section{References}

1. Judge A, Arden NK, Kiran A (2012) Interpretation of patient-reported outcomes for hip and knee replacement surgery: identification of thresholds associated with satisfaction with surgery. J Bone Joint Surg B 94(3): 412-418.

2. Schrier JC, Palmen LN, Verheyen CC, Jansen J, Koëter S (2015) Patientreported outcome measures in hallux valgus surgery. A review of literature. Foot Ankle Surg 21(1): 11-15.

3. Jones S, Hussainy HA, Ali F, Betts RP, Flowers MJ (2004) Scarf osteotomy for hallux valgus. A prospective clinical and pedobarographic study. J Bone Joint Surg Br 86(6): 830-836.

4. Ahmad K, Pillai A, Somasundaram K, Fox A, Kurdy N (2015) Investigating patient-reported outcomes and experience for first metatarsal scarf \pm akin osteotomy for hallux valgus. Bone Joint J 97-B(Supp 14): 23.

5. Suresh SS (2007) Scarf osteotomy-Is it the procedure of choice in hallux valgus surgery? A preliminary report. Oman Med J 22(3): 47-50.

6. Dawson J, Boller I, Doll H (2011) The MOXFQ patient-reported questionnaire: assessment of data quality, reliability and validity in relation to foot and ankle surgery. Foot (Edinb) 21(2): 92-102.

7. Deshpande PR, Rajan S, Sudeepthi BL, Abdul nazir CP (2011) Patientreported outcomes: A new era in clinical research. Perspect Clin Res 2(4): 137-144.

8. Dawson J, Coffey J, Doll H, Lavis G, Cooke P, et al. (2006) A patient-based questionnaire to assess outcomes of foot surgery: validation in the context of surgery for hallux valgus. Qual Life Res 15(7): 1211-1222.

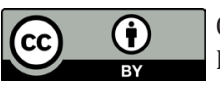

Creative Commons Attribution 4.0

International License

For possible submissions Click Here

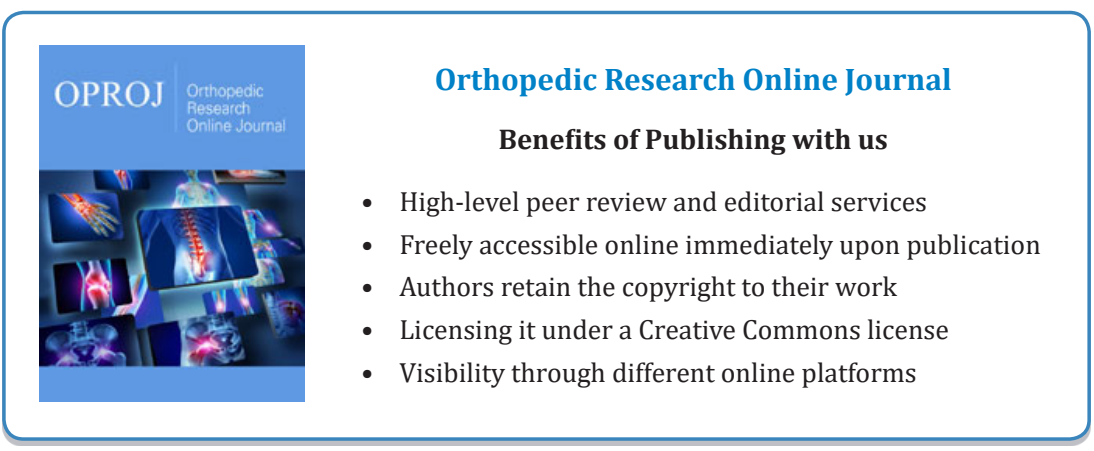

